\title{
A STOCHASTIC VERSION OF THE DYNAMIC LINEAR PROGRAMMING MODEL MESSAGE III
}

\section{S. Messner}

International Institute for Applied Systems Analysis

Laxenburg, Austria

A. Golodniliov

V.M. Glushtiov Institute of Cybernetics

Ii iev, Uhraine

A. Gritsevskii

International Institute for Applied Systems Analysis

Laxenburg, Austria

RR-97-2

January 1997

Reprinted from Energy, Volume 21. No. 9, pp. 775-784, 1996. 
Research Reports, which record research conducted at IIASA, are independently reviewed before publication. Views or opinions expressed herein do not necessarily represent those of the Institute, its National Member Organizations, or other organizations supporting the work.

Reprinted from Energy, Volume 21, No. 9. pp. $775-784,1996$.

Copyright (C)1996 Elsevier Science Ltd.

All rights reserved. No part of this publication may be reproduced or transmitted in any form or by any means, electronic or mechanical, including photocopy, recording, or any information storage or retrieval system, without permission in writing from the copyright holdcr. 


\title{
A STOCHASTIC VERSION OF THE DYNAMIC LINEAR PROGRAMMING MODEL MESSAGE III
}

\author{
S. MESSNER, $† \ddagger$ A. GOLODNIKOV§ and A. GRITSEVSKII $\dagger$ \\ $\dagger$ Environmentally Compatible Energy Strategies (ECS) Project, International Institute for Applied Systems \\ Analysis, A-2361 Laxenburg, Austria and §V. M. Glushkov Institute of Cybernetics, Kiev, Ukraine
}

(Received 4 October 1995)

\begin{abstract}
This paper introduces an approach to modeling the uncertainties concerning future characteristics of energy technologies within the framework of long-term dynamic linear programming models. The approach chosen explicitly incorporates the uncertainties in the model, endogenizing interactions between decision structure and uncertainties involved. The use of this approach for future investment costs of electricity generation technologies in the framework of very long-term energy scenarios shows improvements in model behavior and more robust solutions with respect to technology choices made. Copyright (c) 1996 Elsevier Science Ltd.
\end{abstract}

\section{INTRODUCTION}

Workers at the ECS Project at IIASA are using the dynamic linear programming model, MESSAGE III, for the analysis of long-term energy strategies to mitigate climate change. ${ }^{1}$ MESSAGE III is a technoeconomic optimization model. Other well-known models in this class are, MARKAL, which was developed by the International Energy Agency $\left(\right.$ IEA) ${ }^{2}$ and is mostly used for national energy studies ${ }^{3}$ and EFOM, the model used by the European Union. ${ }^{4}$ Analyses with such models involve the use of information on potential future technology characteristics, energy-service demands and resource availability to investigate paths to a sustainable future energy system.

A major shortcoming of conventional long-term energy-optimization models is the requirement to use point estimates for technology characteristics and other important system parameters. Here, we present an approach to overcome this problem by introducing distribution functions for technology parameters into model formulation. The stochastic version of MESSAGE III captures the risk of underestimating future technology costs. Computational overhead for applying the approach is very low compared with that of the original model; for the runs investigated here, no increase in CPU time was detected. Another problem often encountered with deterministic models, in particular, linear programming models, is their sensitivity to input parameters. Linear programming models are, by definition, the worst in this respect because they tend to favor single solutions and extreme developments instead of mixing various technologies or strategies. In such cases, modelers develop scenarios that are robust with respect to uncertainties in input data. They use parameter variations and delimit the solution space to the area that yields acceptable and robust results. The concept of robustness applied here does not correspond to the concepts found in the literature of energy planning. There, robust solutions should help in the decision making process and should therefore define choices that are relatively attractive under all conceivable conditions. ${ }^{5.6}$ In scenario analysis of potential energy futures, different basic assumptions are expected to define different scenarios. A model is robust if, within a generic scenario, reasonable variations in input data do not generate drastic changes. This variation of input data lies within the variance possible within the scenario. Robust solutions are not expected to stretch over different scenarios. Investigating the effect of data variations within a family of scenarios is rather labor intensive and requires modeling experience. The important parameters for variation have to be found, and model runs with combinations of such parameter variations have to be performed. Such investigations can result in a large number of model runs. Additional constraints that are introduced to stabilize model results reflect expert judgement, which is always subjective to some degree and involves the risk of over or underestimating parameters. Studies on formalized approaches to incorporating uncer- 
tainties and deriving robust solutions have focused on abrupt changes in uncertain exogenous constraints and the required robust strategies, ${ }^{7.8}$ on approaches to exogenously analysing uncertainties using Monte Carlo-based methods such as Latin Hypercube Sampling, 9,10 or on other statistical methods. ${ }^{11.12}$ Considerable effort has been put into improving the methodology applied by electric utilities, where uncertainties concerning demand, resource availability, or environmental constraints imply additional risk in the decision making process. ${ }^{13.14}$

The stochastic approach presented here explicitly introduces uncertainties concerning experts' opinions of future investment costs for technologies. Compared with other approaches, it has the advantage of incorporating the required model additions into the LP formulation, thus avoiding the substantial CPU times required for approaches using exogenous sampling. The data used for the distribution function of investment costs were derived from the greenhouse-gas mitigation-technology inventory called CO2DB and developed by ECS over the last five years. ${ }^{15}$ Some sample technology descriptions from CO2DB are given by Schäfer et al, ${ }^{16}$ while the data and distribution functions used for the current analysis are documented by Wei. ${ }^{17}$

Strategies derived with the stochastic approach possess the required technological diversity without exogenous flexibility constraints. They also have a more robust structure with respect to present uncertainties concerning future parameters than strategies obtained on the basis of deterministic parameters. Moreover, the strategies derived with the stochastic model extension are less costly than strategies obtained using a purely deterministic model.

\section{MODEL DESCRIPTION AND DATA REQUIREMENTS}

MESSAGE III analyses future energy strategies in terms of available technologies, resources, energyservice demands, and pollutant emissions. It is dynamic over time by integrating optimization for the whole time horizon into one objective function and linking different time steps (periods) in the model by various types of constraints.

MESSAGE III has three major types of variables and a variety of equation types. The variables are: (a) technology activity; (b) annual new installations of technologies; and (c) annual resource extraction. The constraints of MESSAGE III can be grouped as follows: (a) demand constraints assuring that the exogenous demand is satisfied by the appropriate technologies; (b) balancing constraints for the energy carriers (e.g. electricity) that guarantee that no more is consumed than is produced; (c) capacity constraints relating production of a technology in a period to the overall capacity existing in the period; (d) dynamic constraints relating the activity in one period to the activity in the previous period; and (e) two types of resource constraints limiting the overall resource consumption to the quantity available and the annual extraction to a fraction of the quantity still available in a period. All variables and most of the constraints can be attributed to one specific time period. Only the dynamic constraints link two time periods to each other; capacity constraints construct the sum of new installations over the plant life of the technology, and the resource constraints are overall constraints that pool the extraction variables into one overall limit.

The simplified formulation of MESSAGE III may be written as follows: $\dagger$

$$
\begin{gathered}
\min \sum_{r=0}^{T}\left\langle C, x^{t}\right\rangle, \\
B_{\imath} x^{t} \geq d^{t}, t=0,1, \ldots, T, \\
\sum_{t=0}^{T} A_{r} x^{t} \leq r, \\
\sum_{k=0}^{t} P_{k} x^{k} \leq e^{t}, t=0,1, \ldots, T, \\
0 \leq x^{t} \leq \overline{x^{t}}, t=0,1, \ldots, T,
\end{gathered}
$$

†For two vectors $x=\left(x_{1}, \ldots, x_{n}\right)$ and $y=\left(y_{1}, \ldots, y_{n}\right)$ the term $\langle x, y\rangle$ denotes $\sum_{i=0}^{n} x_{i} y_{i}$. 
where $C^{\mathrm{v}}=\left(C_{1}, \ldots, C_{\mathrm{n}}\right)$ is the cost vector at the time interval $t=0,1, \ldots, T ; r=\left(r_{1}, \ldots, r_{\mathrm{m}}\right)$ is the vector of overall resources; $d^{n}=\left(d_{1}^{2}, \ldots, d_{\mathrm{c}}^{\mathrm{k}}\right)$ is the vector of energy demands at time $t$ or zero for the energy balances, $e^{t}=\left(e_{1}^{t}, \ldots, e_{1}^{t}\right)$ is the vector of other exogenous right hand sides, e.g. attributable to capacities existing in the base year; $A_{\mathrm{t}}$ is the identity to sum all consumption of one resource over time; $B_{\mathrm{t}}$ is the matrix of the technologies' input/output coefficients; $P_{\mathrm{k}}$ is the matrix providing relations between periods, e.g. the capacity constraints.

MESSAGE III incorporates technical information on technologies, such as efficiency, technical plant life, and pollutant emissions. The objective function used in most applications minimizes the sum of the discounted costs, including the technologies' investment costs and operation and maintenance costs. The costs or profits from international energy trade and from energy or emission taxes can also be considered in the objective function. Technical, social, political, or environmental limitations to the utilization of technologies are represented by several types of constraints. Examples are the quantity of biomass available in a region and the maximum share of wind-power plants tolerable in an electric grid without stability problems. Other constraints are introduced by the modeler to combat the LP models' inherent feature always delivering the cheapest option available to the maximum degree possible. In mathematical terms the model provides a solution that minimizes the objective function and is at an extreme of the solution space. A consequence is that minor changes in cost assumptions can lead to qualitatively different results. This common "flip-flop" behavior is counteracted by the introduction of smoothening constraints, for example, limiting changes over time, limiting new installations of a technology, or linking technologies to each other.

MESSAGE III is applicable to a wide range of energy-related issues such as regional or urban energy planning ${ }^{18-20}$ and to investigations of the future energy system. ${ }^{21-23}$ Its most recent application has been in the joint IIASA/WEC (World Energy Council) study on long-term energy perspectives ${ }^{24}$ for which three families of global energy scenarios for the next century were developed. This analysis relies on assessments of technological characteristics over the coming century, which, due to the nature of the problem and the very long time horizon, are bound to be uncertain.

A major effort in the preparation of the technology data was the development of CO2DB, the IIASA greenhouse-gas mitigation-technology inventory, ${ }^{15}$ which currently covers over 1400 technologies. Of these, approximately 1000 represent various electricity and cogeneration technologies. This extensive database was used to perform a statistical analysis and develop empirical distribution functions of capital costs and other characteristics of future energy technologies. ${ }^{17}$ These distribution functions can be interpreted to encompass energy experts' views, or "conventional wisdom", about future performance characteristics of electricity generation technologies. It should be noted, however, that these estimates of future technology characteristics may not all be independent of each other. Such studies are quite often cross-referenced and there can be considerable variation in performance and cost due to locational and other factors that for obvious reasons cannot be excluded from our statistical analysis.

Performance characteristics of technologies that are mature and widely applied are quite well known. In contrast, for new technologies that currently hold low market shares (e.g. power plants based on combined-cycle technology) or undergo fundamental changes (e.g. nuclear reactors) the range of parameters is considerably wider. For technologies that are not economically (e.g. PV, fuel cells) or even technically viable (e.g. fusion), the funnel of future costs and other characteristics continues to widen due to growing uncertainties.

Of the technologies analysed by Wei, ${ }^{17}$ eight classes were chosen for uncertainty analysis. Table 1 shows the range of investment figures and the arithmetic mean for each of these technologies. The uncertainty of investment cost estimates is considerable for most of them. For coal power plants, the range of estimates varies by US\$1700-1800 per kWe, irrespective of whether conventional or advanced systems are investigated. For the cheaper conventional systems this translates into a $177 \%$ variation; for the capital-intensive advanced systems, such as integrated gasification combined-cycle systems or pressurized fluidized bed combustion, the variation is only $43 \%$. For solar thermal power generation the range is also $50 \%$, but the absolute difference between minimum and maximum estimate is US $\$ 2700 / \mathrm{kWe}$, a value approaching the maximum estimate for advanced coal power plants. Compared with the other options, gas-based systems have low capital costs, which makes the absolute uncertainty rather low, while the relative range is higher than a factor of two and approximately the same as for new nuclear power generation. For solar thermal systems the range of investment estimates is comparable with that of nuclear plants (US $\$ 2500 \mathrm{US} / \mathrm{kWe}$ ), while solar PV are most uncertain with a range EGY $21-9-C$ 
Table 1. Investment-cost range and arithmetic mean for eight new electricity generation technologies [costs are in US(90)\$ $/ \mathrm{kWe}$ ].

\begin{tabular}{l|r|r|r|r|c}
\hline Technology & $\begin{array}{c}\text { Arithmetic } \\
\text { Mean, \$/kWe }\end{array}$ & $\begin{array}{c}\text { Minimum, } \\
\$ / \mathrm{kWe}\end{array}$ & $\begin{array}{c}\text { Maximum, } \\
\$ / \mathrm{kWe}\end{array}$ & $\begin{array}{c}\text { Range, } \\
\$ / \mathrm{kWe}\end{array}$ & $\begin{array}{c}\text { Range, } \\
\text { max./min. }\end{array}$ \\
\hline Conventional Coal & 1350 & 650 & 2450 & 1800 & 2.77 \\
Advanced Coal & 1695 & 1195 & 2905 & 1710 & 1.43 \\
Conventional Gas & 570 & 330 & 1050 & 720 & 2.18 \\
Gas Combined-Cycle & 815 & 514 & 1702 & 1188 & 2.31 \\
Biomass Power Plant & 1580 & 500 & 3020 & 2520 & 5.04 \\
Nuclear Electricity & 2145 & 1070 & 3600 & 2530 & 2.36 \\
Solar Thermal Electricity & 3010 & 1790 & 4490 & 2700 & 1.51 \\
Solar-PV Electricity & 6120 & 1740 & 12540 & 10800 & 6.21 \\
\hline
\end{tabular}

of more than US $\$ 10,000 / \mathrm{kWe}$. For all estimates the arithmetic mean of estimates lies below the middle of the range covered.

The model analysis is based on the global energy model used for the joint IIASA/WEC study. ${ }^{24}$ The model developed for this study consists of 11 regional models covering the world energy system. Energy flows include all relevant energy carriers and conversion technologies from coal mining and oil drilling via various electricity-generation options to final consumption, e.g. in industrial boilers or in road transport. The study's time-frame is to the year 2100 . For the current analysis a compressed version of this global energy model is applied. It aggregates the 11 world regions into one region but includes all technological detail concerning electricity generation from the more detailed regional approach. The time-frame is to the year 2050 .

Figure 1 shows the sensitivity of electricity generated from gas combined-cycle power plants to variations in investment costs of this and other technologies for the year 2050. The straight line at $100 \%$ represents the initial model run using the arithmetic mean as investment costs for the new technologies (here labeled arithmetic mean). In addition, two types of sensitivity runs were performed: the low-cost cases reduce investment costs of one specific technology or all new technologies (labeled "ALL") to the minimum estimate from Table 1; the high-cost cases use the maximum estimates. The results, as displayed for the gas combined-cycle technology, show that sensitivity is very high to the

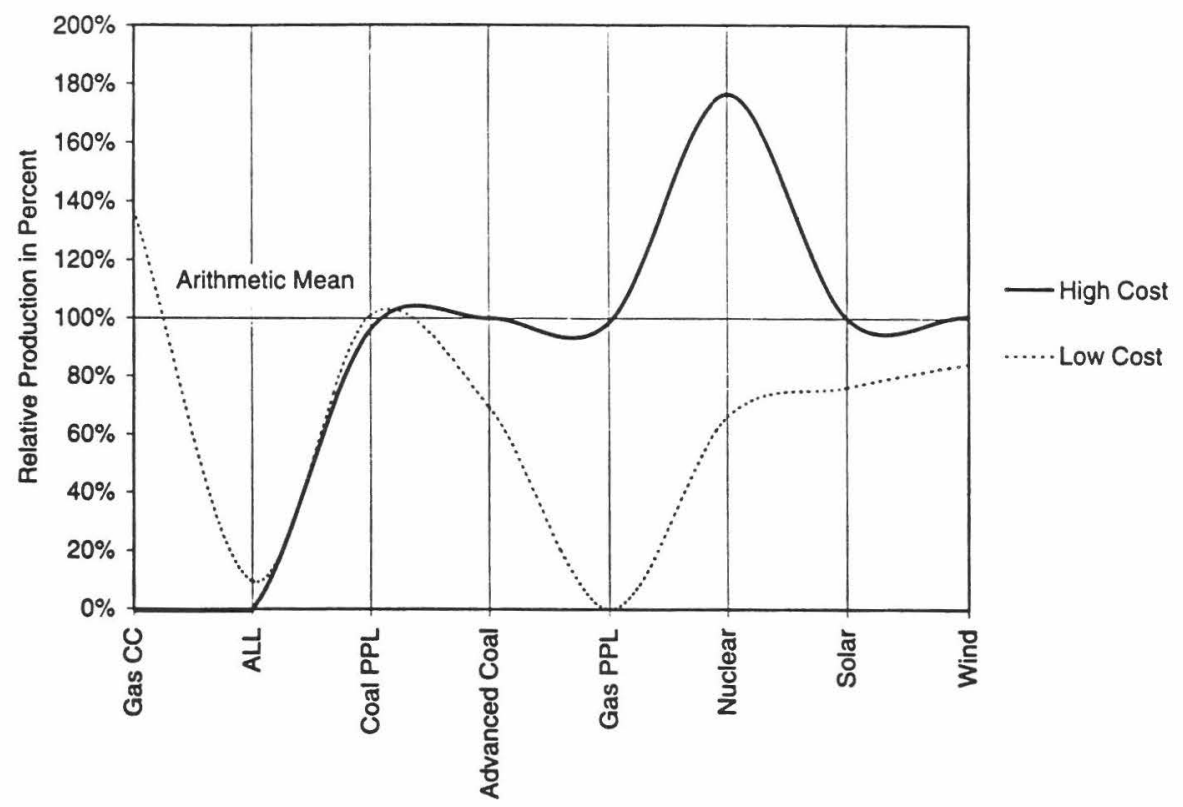

Fig. 1. Electricity production of gas combined-cycle systems for the year 2050 as a function of varying cost assumptions for the other technologies, relative to the arithmetic mean case. 
own cost estimates of the technology: high costs reduce the use to zero and low costs increase it by another $40 \%$ compared with the arithmetic mean. If all investments are changed at once (i.e. taking either all low or all high ends of the range), the gas combined cycle is a loser, either because at least one of the others becomes cheaper (low cost) or because the combined cycle is too expensive (high cost). In all cases where other single technologies are at the low end of cost estimates the gas combinedcycle system is reduced between 15 and $30 \%$; the only case showing major gains is when new nuclear reactors become expensive.

The extreme sensitivity of the model results to admittedly very high cost variations points to the weakness of the deterministic cost-minimization approach: in order to find strategies that are resilient with respect to parameter changes, a multitude of model runs would be required to further investigate synergies and competition of the technologies, and to locate more exactly the investment cost points at which the model starts to flip into another stratum of the energy system. The approach suggested in the next section enables the modeler to perform such analyses in a closed form by incorporating the uncertainties and risk concerning future investment costs of the technologies into the mathematical formulation of the model.

\section{INCORPORATING UNCERTAINTIES}

In the stochastic application of MESSAGE III the vectors $C$ described in the previous section are treated as random vectors. We define them as $C(w)$, where $w$ is an element from a probability space indicating the dependence of the cost vector $C^{\mathrm{k}}(w)$ on a random event that is characterized by a probability measure $d P(w)$. This measure may be derived from observations or from expert judgements. We assume that the initial distributions at $t=0$ are as given in $\mathrm{Wei}^{17}$ and described in the previous section.

If the cost vector $C^{\mathrm{k}}$ is stochastic, then the cost $\sum_{\mathrm{t}=0}^{\mathrm{T}}\left\langle C(w), x^{\mathrm{l}}\right\rangle$ of a given strategy $x=\left(x^{0}, \ldots, x^{\mathrm{T}}\right)$ can be derived from the probability distributions of $C(w)$. The underestimation of the expected cost incurred by using the deterministic model is calculated as follows. For a given strategy $\left\{x^{t}\right\}, t=0,1$, $\ldots, T$ and an observed scenario $w$ of the cost path $\left\{C^{T}(w)\right\}, t=0,1, \ldots, T$, the positive deviation of the observed total cost $\Sigma_{t=0}^{\mathrm{T}}\left\langle C(w), x^{t}\right\rangle$ from the calculated cost $\Sigma_{t=0}^{\mathrm{T}}\left\langle C^{t}, x^{t}\right\rangle$ is defined as

$$
\sum_{r=0}^{T} \max \left\{0,\left\langle C^{(}(w)-C, x^{t}\right\rangle\right\},
$$

where

$$
\max \left\{0,\left\langle C^{(}(w)-C, x^{t}\right\rangle\right\}=\max \left\{0, \sum_{j=1}^{n}\left(C_{\mathrm{j}}^{(}(w)-C_{\mathrm{j}}\right) x_{\mathrm{j}}\right\} .
$$

Let us denote this deviation as $R(x, w)$ for strategy $x=\left(x^{0}, x^{1}, \ldots, x^{\mathrm{T}}\right)$. This is an expression of the underestimation of the costs for the strategy $x$ using the deterministic cost function. The expected cost of underestimating $R(x)=\mathbf{E} R(x, w)$ can be used as an indicator of the economic risk associated with strategy $x$. The risk function $R(x)$ can be taken as an additional nonlinear constraint to the original deterministic model, or it can enter the objective function as an additional "penalty" term. An extensive discussion of motivation, formulation, and solution procedures for the optimization of functions, expressed in terms of expectations similar to $R(x)$, is given by Ermoliev and Wets. ${ }^{25}$

Applying the second alternative, using the "penalty" term, the stochastic model that explicitly takes into account the risk of underestimating future investment costs can be formulated as minimizing

$$
F(x)=\sum_{i=0}^{T}\left\langle C, x^{t}\right\rangle+\rho R(x)
$$

subject to the original constraints [Eqs. (2-4)]. It is also possible to add some or all constraints [Eq. (5)]. Choice of decisions under uncertainty is analysed by many authors on the basis of the mean and variance of gains or losses. ${ }^{26.27}$ The proposed model also incorporates this type of analysis. It uses directly available information on monetary losses and does not incorporate social values of losses expressed as utility functions. The function $R(x)$ reflects asymmetric variances of losses, the non-negative parameter 
$\rho$ represents risk aversion similar to the mean-variance analysis described by Pratt. ${ }^{26}$ The case where $\rho=0$ corresponds to risk neutrality. When $\rho=1$ the first term of $F(x)$ corresponds to the expected cost associated with the energy developments and the second term corresponds to the expected underestimation of the cost. Applying a risk factor $\rho>1$ emphasizes the risk (risk aversion), whereas $\rho<1$ reflects a tendency toward risk neutrality.

It is possible to impose additional constraints on the level of risk $R(x)$ :

$$
R(x) \leq \bar{R},
$$

where $\bar{R}$ is an upper bound on the underestimation of the costs. In a sense the parameters $\rho$ and $\bar{R}$ regulate the robustness of a strategy $x$ with respect to the uncertainties. Here the trade-off is between robustness and cost: the more robust a strategy is with respect to potential changes in system parameters, the higher the cost of implementing this strategy will be.

The resulting stochastic optimization problem is solved on the basis of simultaneous approximation of $R(x)$ by $N$ sample functions:

$$
R(x) \sim \frac{1}{\mathrm{~N}} \sum_{s=1}^{N} R\left(x, w^{\mathrm{s}}\right)
$$

where $w^{1}, \ldots, w^{\mathrm{s}}, \ldots, w^{\mathrm{N}}$ are independent simulations ("scenarios") of possible cost paths $\left\{C\left(w^{\mathrm{s}}\right)\right\}$, $t=0,1, \ldots, T$ and $\mathrm{s}=1,2, \ldots, N$. The performance function $F(x)$ is then approximated by including the sequence of random functions:

$$
F^{\mathrm{N}}(x)=\sum_{t=0}^{T}\left\langle C, x^{t}\right\rangle+\rho \frac{1}{\mathrm{~N}} \sum_{s=1}^{N} R\left(x, w^{\mathrm{s}}\right) .
$$

The solution path of the optimal strategies $\left\{x^{N}\right\}$ with $N \rightarrow x$ that are derived from optimization of the functions $\left\{F^{\wedge}(x)\right\}$ converges to an optimal solution minimizing Eq. (6).

The model is solved for a sufficiently large $N$, where the size of $N$ is evaluated by experiments. Several successive model runs with the same $N$ are compared; if no major changes in the solutions occur, $N$ is sufficiently large. It would also be possible to design an iterative sampling procedure to derive the optimal size of $N$.

\section{RESULTS OF NUMERICAL EXPERIMENTS}

As described in Sec. 2, the model used for this analysis is based on a large-scale, long-term energy model developed for the IIASA/WEC analysis of the global energy system. ${ }^{24}$ The 11 regional energy models combined into a world model were developed by a team of experts in a cooperative effort. Constraints were used to derive realistic, perhaps more costly, strategies involving combinations of technologies and to represent technical, social, or other limitations on the energy system. An example of such a limitation is to constrain solar and other renewable sources of electricity with stochastic availability to a maximum share of the total electricity generated. In the IIASA/WEC analysis the model was used to quantify six partly very diverging scenarios. These scenarios were modeled with minimum intervention, i.e., only model parameters belonging to the scenario definitions, such as demands or available resources, were changed. The wide range of futures expressed with the model is an indication that the model is not overconstrained. When assumptions are changed, the model outcomes change. The compressed version of the global energy model used here relies on the same data base as the large model and was calibrated to this model, mainly by additional constraints emulating the greater degree of detail in the large model.

To study the performance of the stochastic version of MESSAGE III, three cases were analysed: the deterministic bounded case, where the solution structure of the deterministic model is regulated (bounded) by additional exogenous constraints on the decision variables, was the basis of the analysis. This case corresponds to the arithmetic mean case in Sec. 2. For the deterministic unbounded case the exogenous constraints to guide the deterministic model were not included in the model, and in the stochastic unbounded case the stochastic performance function was added to the deterministic unbounded model. 
This choice of cases was influenced by the problem analysis in Sec. 2, where one of the shortcomings of a deterministic approach using MESSAGE-type models was identified as requiring additional constraints to make the model more robust. The model of the arithmetic mean case, referred to here as the deterministic bounded case, is relaxed in terms of the exogenous constraints on the investigated technologies. This model with a larger solution space, the deterministic unbounded case, shows where the modelers have applied their expert judgement in order to constrain the solution toward the direction they think more appropriate and to gain results that are more resilient to parameter changes. Based on this case, the stochastic model was used to investigate strategies that minimize the risk of cost overrun due to the uncertainties in investment costs.

Figures 2 and 3 show the development of coal and gas-based electricity generation for the three cases to the year 2050. One of the reasons for the limitations in the arithmetic mean case is that the effects of present policies and planned installations are incorporated this way. Figures 2 and 3 indicate that in the deterministic bounded case more coal and less gas is used for electricity generation in the nearer future than in the other cases. This reflects the regulations that were or still are applied in many European countries, $\dagger$ the strong emphasis that international organizations such as the IEA put on coal-based electricity generation, and a general reluctance of utilities to use natural gas for electricity generation. In the unbounded cases this constraint is relieved and leads to an immediate substitution of natural gas for coal. In future applications, constraint relaxation will have to be related only to the more distant future and limits on the nearer future should remain intact.

The general trend for all model applications is similar, with coal use declining and gas use increasing over time. However, the deterministic unbounded case uses the most gas, with a strong swing around 2030. This swing is reduced in the deterministic bounded case and practically avoided in the stochastic model. This generally leads to reduced gas use toward the end of the horizon, and, in the deterministic unbounded case, to a rebound of coal-based power generation. This rebound can be avoided in the deterministic bounded and the slochastic cases. The main reason for the generally smoother curves in the stochastic case compared with the other cases is that the approach avoids running into short-term optima for single time steps. The stochastic approach helps generate strategies where more technologies

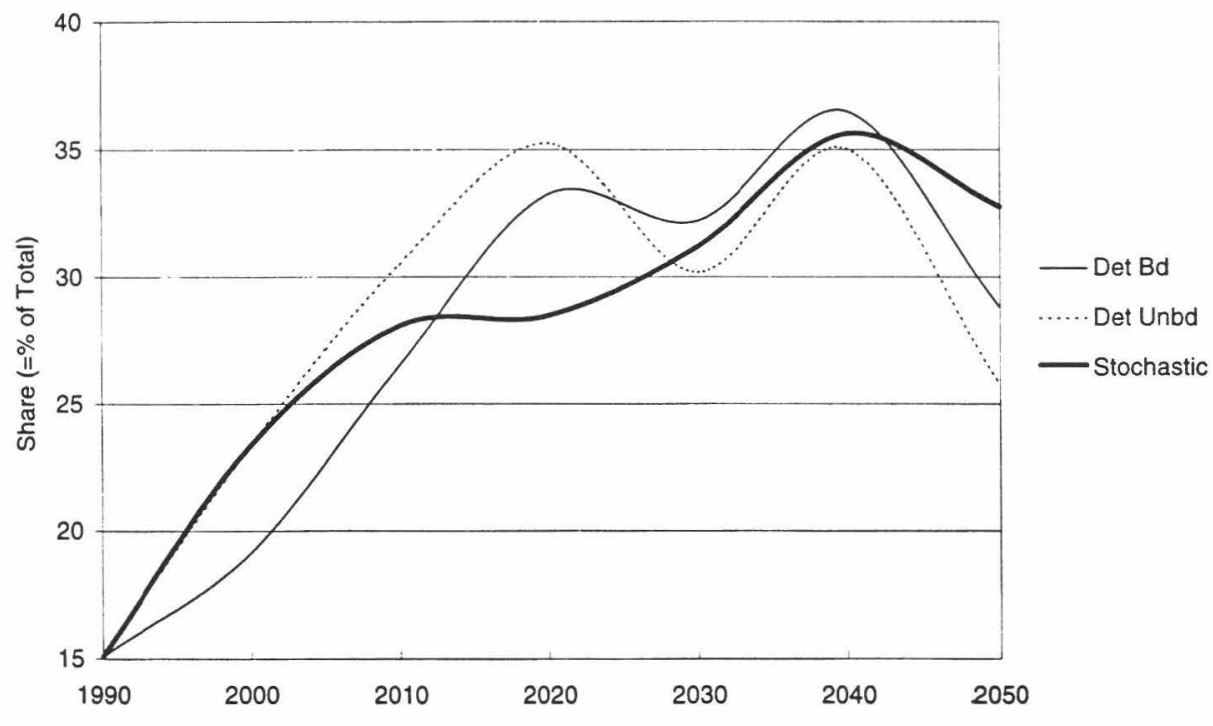

Fig. 2. Electricity generation from gas for the deterministic bounded (Det Bd), deterministic unbounded (Det Unbd) and stochastic cases as share of the total.

† Germany still supports the use of domestic coal in power generation by a subsidy system. In the UK power generation is contractually bound to domestic coal production despite the privatization of the industry. ${ }^{28}$ 


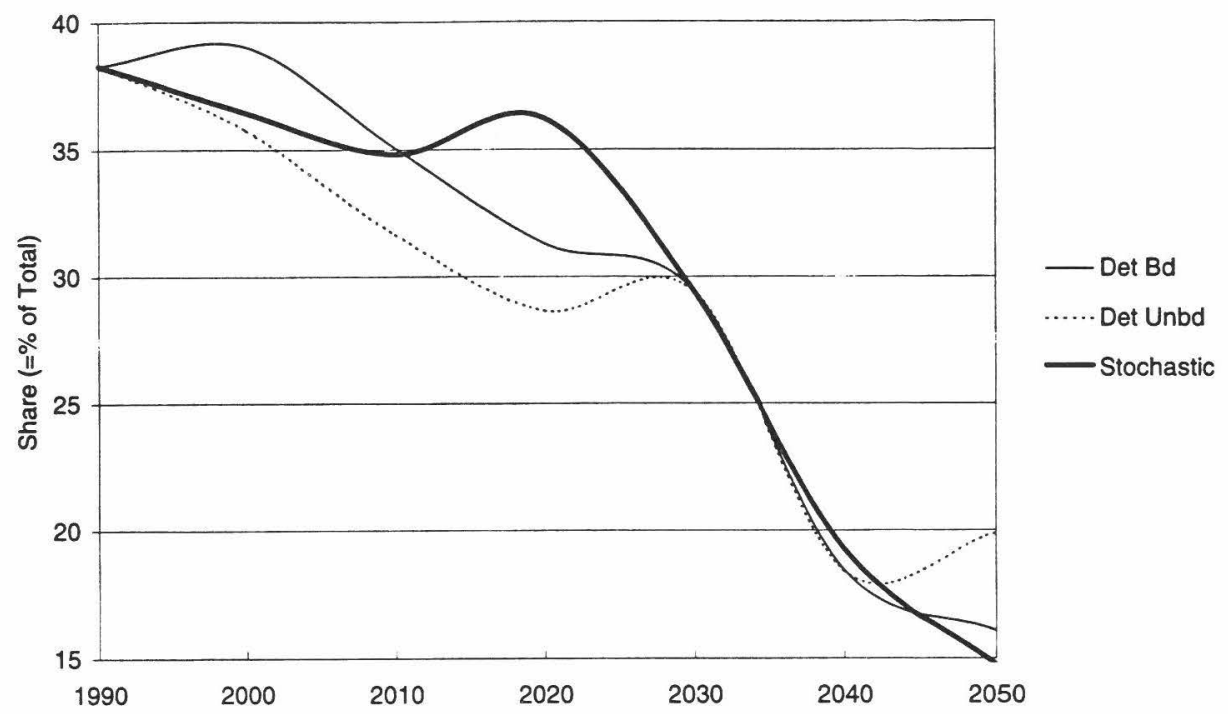

Fig. 3. Electricity generation from coal for the deterministic bounded (Det Bd), deterministic unbounded (Det Unbd) and stochastic cases as share of the total.

are involved and general trends are not neglected to obtain short-term cost reductions. This neglect is exemplified by the two very distinct peaks in gas-based electricity generation in Fig. 2.

Figure 4 compares electricity generation by technology for the year 2050. In the deterministic cases the use of conventional coal power plants and, to a lesser degree, nuclear power plants is reduced in the bounded case compared with the unbounded case. On the other hand, gas-fired combined-cycle power plants are used less in the unbounded model. Introducing the stochastic model formulation gives an interesting result: the tendencies enforced in the bounded versus the unbounded deterministic case are supported by the model outcomes. Gas combined cycles are applied to an even higher degree than in the deterministic bounded case and coal-based power generation and nuclear reactors supply even less electricity. This result suggests that in the deterministic bounded case the modeler, anticipating the

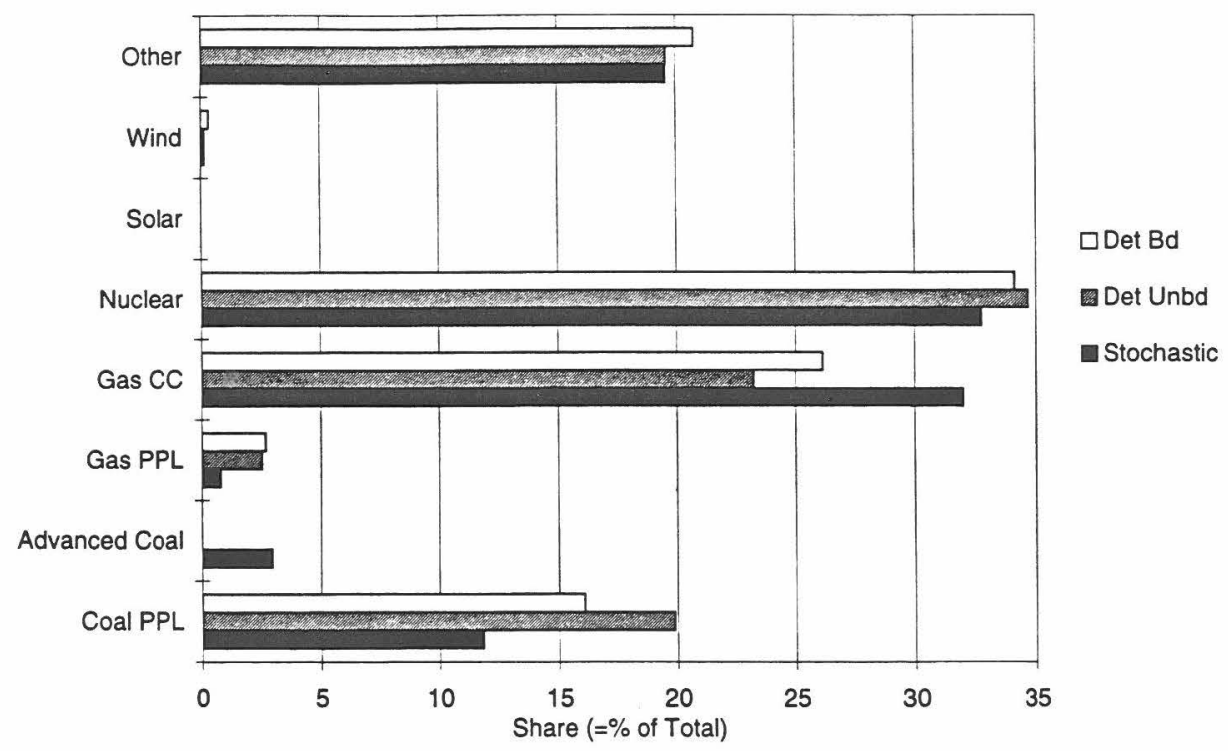

Fig. 4. Electricity generation by technology for the deterministic bounded (Det Bd), deterministic unbounded (Det Unbd) and stochastic cases in 2050 as share of the total. 
uncertainties concerning future developments of model parameters, chose bounds to minimize the risk of selecting the wrong technology. In the stochastic unbounded case these modeler's choices are applied in an even more pronounced manner.

The split between the two different systems for coal-based and gas-based electricity generation deserves special attention. In the case of coal-based systems, i.e. conventional and advanced coal power plants, the cheaper and slightly less efficient conventional systems are preferable. If the uncertainties of investments are incorporated, a diversification takes place and some $20 \%$ of the coal-based systems are advanced systems. For gas-based systems, the effect is different: the new systems, combined cycles, are already preferable in the deterministic cases. The stochastic case has two effects: it sharply increases the application of combined-cycle technology and it reduces the use of conventional steam-based power generation from gas. This shows that the gas combined cycle has the most attractive cost structure, even with uncertainties of future investment costs. The reason lies in the distribution of investment costs of gas combined cycles: $30 \%$ of the estimates are at the lower end of investment figures, around US\$500/kWe. Clearly the majority of experts envisage gas-based combined-cycle systems to be very competitive technologies.

Finally, one general conclusion is attributable to the approach taken. Because the diversification in the stochastic model is obtained in a "natural" way, without constraining model flexibility, there is a high probability that the overall cost of the optimal strategy derived by the stochastic model will be lower than in the deterministic model. Hard limits could also eliminate desired solutions at any time whereas incorporating additional information in the objective function leaves the model the flexibility to choose good solutions.

\section{CONCLUSIONS AND OUTLOOK}

The goals of this paper were to introduce the stochastic version of MESSAGE III and to analyse and compare structures of energy development strategies derived from the deterministic and stochastic versions. The stochastic version deals with uncertainties concerning future investment costs by incorporating the expectation of incurring higher costs due to these uncertainties into the objective function.

The first, rather striking result of the experiments is that skilled model application of a deterministic model yields results with diversification features comparable with those of the stochastic version. By using iterative model adaptations and analysing the result, experienced modelers will reach results displaying adequate robustness with respect to the model parameters.

However, the results of the numerical experiments also show several advantages of the stochastic model. In stochastic models the diversification of development strategies is reached in a natural way as a result of an anticipation of possible variations rather than as a result of additional flexibility constraints. Consequently, diversification in the stochastic case tends to be less "expensive" than diversification through flexibility constraints. In the described application, the optimal value of the objective function in the stochastic model is lower than the optimal value of the objective function in the bounded deterministic model.

Applying the stochastic approach to energy strategy evaluation leads to a shift of energy production from technologies with high sensitivity to parameter changes toward technologies with less sensitivity, even if they are more expensive in some cases. A consequence of this and the inherent minimization of risks related to singular development paths is early diversification into new technologies.

Uncertainties concerning future technology characteristics clearly do not concern economic indicators alone. A prime candidate for further investigations is technical performance, primarily expressed by conversion efficiency and accompanying pollutant emissions. Distribution functions for efficiency estimates of electricity generation technologies are available in the same fashion as the investment cost functions and could be readily incorporated into an extended stochastic version of MESSAGE III. Moreover, other parameters, such as the date of introduction of new technologies and perhaps the cost and performance effects of "learning by doing" (so-called technological learning), ${ }^{29}$ are of interest for future investigations.

Leaving aside the representation of technologies in MESSAGE III, this model incorporates many more constraints where experts' opinions diverge, such as resource quantities available worldwide or limits on $\mathrm{CO}_{2}$-emissions required to mitigate global warming. The developments of stochastic approaches covering these cases are challenging from a methodological point of view, especially taking into account the large-scale applications of MESSAGE III. 
An important direction of further studies concerns the development of generators for dependent random parameters with a possible reduction of uncertainties. In our study we ignored dependencies between variations of investment costs for a technology and, for example, total cumulative investment in this technology. On the other hand, economic recession or an increase of economic activity affects all activities and shows the correlation between changes in time and between various types of model parameters. The analysis reported in this paper could serve as a basis for possible approaches to investigating such situations in a more realistic manner.

Acknowledgements - This paper is a result of collaborative work of the ECS and RPC Projects at IIASA. The authors are grateful to Y. Ermoliev, A. Grübler, N. Nakićenović, and an anonymous referee for helpful and constructive discussions and suggestions.

\section{REFERENCES}

1. S. Messner and M. Strubegger, "User's Guide for MESSAGE III", WP-95-69, IIASA, Laxenburg, Austria (1995).

2. L. G. Fishbone, G. A. Giesen, H. A. Goldstein, H. A. Hymen, "User's Guide for MARKAL (BNL/KFA Version 2.0)", BNL-46319, Brookhaven National Laboratory, Upton, NY (1983).

3. T. Kram, "National Energy Options for Reducing $\mathrm{CO}_{2}$ Emissions", Report of the Energy Technology Systems Analysis Programme, Annex IV (1990-1993), ECN-C-93-101, Netherlands Energy Research Foundation ECN (1993).

4. E. Van der Voort, E. Donni, C. Thonet, E.Bois d'Enghein, C. Dechamps and J. F. Guilmot, "Energy Supply Modeling Package EFOM-12C MARK I", Cabay, Brussels (1984).

5. J. M. Mulvey, R. J. Vanderbei and S. A. Zenios, Operations Research 43, 264 (1995).

6. J. A. Bloom, Operations Research 31, 84 (1982).

7. T. Larsson and C-O, Wene, International Journal of Energy Research 17, 503 (1993).

8. A. S. Manne and R. G. Richels, " $\mathrm{CO}_{2}$ Hedging Strategies: the Impact of Uncertainty upon Emissions", in The Economics of Climate Change, Conference Proceedings, OECD/IEA, Paris (1994).

9. A. Ford and M. D. McKay, Energy Systems and Policy 9, 217 (1985).

10. G. Infanger, "Monte Carlo (Importance) Sampling within a Benders Decomposition Algorithm for Stochastic Linear Programs. Extended Version: Including Results of Large-Scale Problems", Technical Report SOL 961, Systems Optimization Laboratory, Stanford University, CA (1991).

11. R. L. Iman and J. C. Helton, Risk Analysis 8, 71 (1987).

12. P. E. Love, in Energy Modeling and Simulation, pp. 195-200, A. S. Kydes, A. K. Agrawal, S. Rahman, R. Vichnevetsky and W. F. Ames eds, North-Holland, New York (1983).

13. F. Louveaux and Y. Smeers, in Modeling of Large-scale Energy Systems, pp. 313-320, W. Häfele and L. K. Kirchmayer eds, IIASA proceedings series; vol 12, Pergamon Press, Oxford (1981).

14. B. F. Hobbs, J. C. Honious and J. Bluestein, IEEE Transactions on Power Systems 9, 167 (1994).

15. S. Messner and M. Strubegger, "User's Guide to CO2DB: the IIASA $\mathrm{CO}_{2}$ Technology Data Bank-Version 1.0", WP-91-31a, IIASA, Laxenburg, Austria (1991).

16. A. Schäfer, L. Schrattenholzer and S. Messner, "Inventory of Greenhouse-Gas Mitigation Measures. Examples from the IIASA Technology Data Bank", WP-92-85, IIASA, Laxenburg, Austria (1992).

17. Z. Wei, "Economic Cost Evaluation of Power Generation Technologies", IIASA, Laxenburg, Austria (forthcoming).

18. S. Messner, "Beispiel einer modellgestützten Analyse eines städtischen Energiesystems am Beispiel Stockholm", in Globale und nationale Probleme der wissenschaftlich-technischen Strategienbildung für das Energiesystem, Akademie der Wissenscharften der DDR, Kolloquien, Heft 44, Berlin (1984).

19. R. Pfaff, O. Loibl, S. Messner and M. Strubegger, Österreichische Zeitschrift für Elektrizitätswirtschart 44, 170, Vienna (1991).

20. S. Messner and M. Strubegger, "Energy Policy Evaluation for Cities", in Proceedings of the International Conference on Energy and Environment, Begrell House, NY (in press, 1996).

21. S. Messner and M. Strubegger, "First-Order Effects of a Nuclear Moratorium in Central Europe", WP-86-80, IIASA, Laxenburg, Austria (1986).

22. N. Nakićenović and S. Messner, "Solar Energy Futures in a Western European Context", WP-82-126, IIASA, Laxenburg, Austria (1982).

23. S. Messner and M. Strubegger, Energy-The International Journal 16, 1379 (1991).

24. A. Grübler, M. Jefferson, A. McDonald, S. Messner, N. Nakićenović, H-H. Rogner and L. Schrattenholzer, "Global Energy Perspectives to 2050 and Beyond", WEC, London, U.K. (1995).

25. Y. Ermoliev and R. Wets eds, Numerical Techniques for Stochastic Optimization, Computational Mathematics, Springer, Heidelberg (1988).

26. J. W. Pratt, Econometrica 32, 122 (1964).

27. H. Markowits, Portfolio Selection, Wiley, New York (1959).

28. F. Oosterhuis, Energy Studies Review 6, 226 (1994).

29. K. Arrow, Review of Economic Studies 29, 155 (1962). 